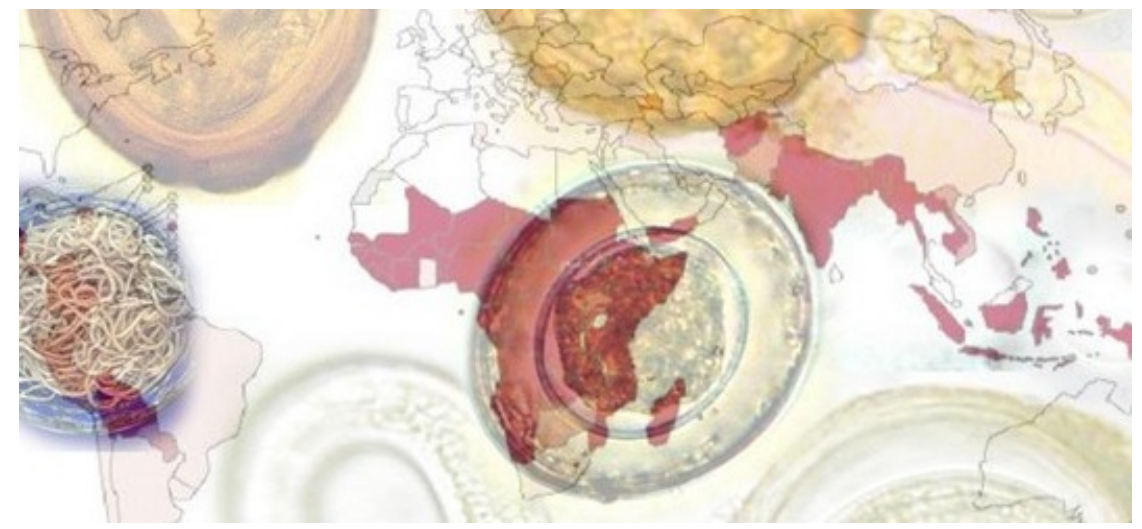

\title{
The global helminth belt and Covid-19: the new eosinophilic link
}

\author{
Carlos Rodriguez \\ 1 Fundación Jiménez Díaz
}

Funding: The author(s) received no specific funding for this work.

Potential competing interests: The author(s) declared that no potential competing interests exist.

\section{Abstract}

This paper expands on our previous report of a striking fact: the low prevalence of the Covid-19 disease in tropical and subtropical areas of the world coincides with the areas of global high prevalence of helminth infestation. In the former paper, we asked if this could be due to a predominant immune phenotype in the populations chronically infected by helminth parasites. We then quoted literature that confirms the high eosinophil counts in the general population of helmint prevalent regions. Now, in this paper, we delve into the different biological mechanisms behind this observation and describe how chronic helminth infection closely correlates with a type of immune phenotype that is characterized by high levels of eosinophils and a stifled inflammatory response. We also argue that this phenotype is the perfect setting to avert both the infection with the Covid-19 virus and the Acute Respiratory Distress Syndrome (ARDS) that is the main cause of death from the disease. In this model, the viral infection would be prevented mainly by the action of the overrepresented eosinophilic cells with strong activity against RNA-virus like the SARS-CoV2. On the other hand, the basal low immune reactivity, as present in chronic helminth infected people, would make very 
unlikely the cytokine storm that is at the core of the development of ARDS. Our main hypothesis therefore is that chronic helminth infection, and the immune consequences thereof, is the main reason why the Covid-19 pandemic have a relatively much lower presence in the millenarian global helmith belt than in the modern urban "dewormed" world.

This model has important implications to 1) the way we deal with the pandemic in the countries of the helminth belt and 2) to design or repurpose drugs that promote the activation of the Th2 immune system and the eosinophils thereof.

\section{INTRODUCTION}

In a previous post of April 27th, we advanced a compound theory that explains why allergic asthma and atopia are protective factors against the Covid-19 disease. Our main conclusion was that, in allergic people, there is a shift towards a predominant Th2 type of immune response. In that paper, we also suggested that this immune signature would probably be the reason why modifiers like living in areas with high helminth prevalence or being an active smoker ${ }^{[1][2]}$ could paradoxically be protective against Covid-

19, given that both preconditions have one thing in common: an activated Th2 immune system $^{[3]}$ and a high eosinophil count.

\section{The Eosinophilic link in Allergic Diseases}

With a big brush, the Th2 immune system is characterized by the presence of predominantly attenuated tissue damage responses and accentuated tissue repair mechanisms ${ }^{[4][5][6]}$. This can be fittingly represented by the overwhelming presence in allergy subjects of two types of cells that are the main workforce of the Th2 phenotype: 1) the Alternatively Activated M2 macrophage, a type of macrophage that is less inflammatory and more reparative than the classical M1 type (the one involved in the first wave of immune defense against foreign invaders) ${ }^{[7]}$, and 2) the eosinophil, a type of terminally differentiated granulocyte cel|[ ${ }^{[8]}$ that, besides being a principal agonist in the pathophysiology of asthma ${ }^{[9]}$, is also a powerful anti-viral cell ${ }^{[10][11]}$, equipped with what is surely the best weapon arsenal that a single cell can have against RNA viruses (viruses like the one causing the Covid-19 disease). Indeed, the hallmark of Eosinophils is the overwhelming presence in their cytoplasm of a type of granules, the so called specific eosinophilic granules ${ }^{[12][13]}$, that are fully loaded with two molecules (the RNAses EDN and ECP), that are tailor made to annhilate RNA viruses ${ }^{[14][15][16]}$. That a single cell can have such a specialized feature is unique in nature and speaks volumes of the role of eosinophils as potential first line defense cells in the fight against viruses like the SARSCov2 virus ${ }^{[17][18][19] . ~}$ 


\section{Helminths}

Helminths, or worms, are invertebrate animals that comprise a whole range of different pathogens able to affect human health. These parasites are included under two major phyla: the nematodes (or roundworms) and the platyhelminthes (also known as flatworms), the latter in turn being subdivided into trematodes (flukes) and cestodes (tapeworms).

Some of them like hookworms, ascaris lumbricoides, or trichiura trichiuris have an oralfecal mechanism of transmission while others like hookworms or Ancylostomas find their way into the host's blood vessels through the exposed skin. Others, like the wurchereria bancroftithat causes lymphatic filariasis or the onchocerca volvulus causing the riverblindness disease, are transmitted through permissive diptera. Finally, some helminths, like the T richinella spiralis are acquired by ing estion of contaminated meat ${ }^{[20][21]}$.

Although helminth parasitic infections have been largely eliminated in developed countries (due to control of the insect vector population by the safe disposal of human waste and the availability of efficient drugs), helminths still infect a vast number of people all over the world, and it is estimated that soil-transmitted helminths alone, cause infection in more than 1.5 billion people, or $20 \%$ of the entire human population ${ }^{[22]}$. As we will see in the map section of the paper, the human population exposed to helminth infection is mainly concentrated in the so called economically disadvataged and/or developing world, including some South and Central American patches, most of tropical and subtropical Africa as well as Southern Asia, in what we call the global helminth belt ${ }^{[23][24]}$. Most, if not all, of the countries therefrom are heavily hit either by poverty, poor sanitation, low access to minimal health services or by all of them together[25][26][27].

Immune response to helminths and the global eosinophilic belt Parasites are extremely adept to moulding themselves to the environment and, most importantly, to evading the host defense system. Over the ages metazoan parasites have learned to survive for very long periods within the bloodstream, lymphatics, liver, or the gastrointestinal tract of their hosts resulting in pathologic conditions such as anemia, cirrhosis, and lymphatic filariasis ${ }^{[28]}$.

Upon a first encounter with helminths, human tissues react as they would against any other foreign invader (be it virus, bacteria, molds or protozoans). In those tissues serving as doorway to the parasite (lung or intestinal mucosae, skin), there is an immediate immune reaction that begins with the activation of the so called innate 
immune system. This system is present in most exposed tissues of higher organisms and is akin to a "face recognition system", by which foreign molecules are immediately spotted by pattern recog nition receptors on epithelial cells and some cells of the immune system. Upon identification of alien molecules, the system's alarms go off in the form of molecular cascades leading to the rapid recruitment of inflammatory cells to the site of the invasion ${ }^{[29][30]}$. Ag ainst common unicellular pathogens like bacteria or viruses, this reaction is characterized by the activation of a Th1 type of immune response that includes the production of proinflammatory cytokines (TNF $\alpha$, IL-1 $\beta$, IFNY IL-12, etc.) and the classical activation of M1 macrophages involved in pathogen killing and phagocytosis. With higher multicellular parasites like helminths, however, it is a whole different story. In fact, most helminths find a way, through different molecular tricks, to avoid the fearful Th1 immune reaction and, instead, invoque the activation of the Th2 network, a completely distinct reaction of the immune system with often opposite effects to the Th1 activation ${ }^{[31]}$.

The ways parasites orchestrate such a response are diverse ${ }^{[32]}$ and hold a common purpose: to promote the symbiotic survival of both parasite and host. In order to achieve this goal, upon infection, most parasites release products that have enzymatic activities to modify both parasite and host molecules leading to either degradation of antiparasitic molecules ${ }^{[33][34]}$ or to the inactivation of the innate immune system ${ }^{[32][35]}$. This usually results in the production of alternative cytokines (IL-4, IL-5 and IL-13) instead of the classical proinflammatory hormones (TNF $\alpha$, IL-1 $\beta$, IFN $Y$ ) of the activated Th1 response. Therefore, the new cytokines trigger the mobilization of a whole array of different cells (Th2 lymphocytes, IgE producing B cells, Mast cells, Basophils, Eosinophils and M2 macrophages) as well as molecules that altogether result in a more lively environment for the parasite and a manageable damage for the host (in the form of some tissue lesion, granuloma formation, fibrosis, etc. depending on each type of parasite).

Importantly, in the so called chronic state of infection, parasites eng ineer strategies to maintain the host in an immune "silent" state. Most in the field agree that parasitic helminths (worms) modify the immune system of their host to avoid immune ejection, a strategy that promotes their long-term survival and results in chronic infection, but that also has a bystander positive effect by protecting both the host and the parasite against the onset of inflammatory disorders that result from a dysregulated immune response $^{[36][37][38]}$. Perhaps the best example of that scheme is wuchereria bancrofti, the helminth that causes lymphatic filariasis. Wuchereria secretes a phosphorylcholine 
moiety (called ES-62) that does it all. Upon secretion, ES-62 inhibits the production of proinflammatory cytokines like IFN- $\gamma$, suppresses antibody production by blocking the interaction between CD4+ cells and B cells, and activates the production of the antiinflammatory cytokine IL-10 and stifles the Th1 pathway [39][40]. Its anti-inflammatory activity is so powerful that it has been proposed as a treatment for auto-immune diseases and even for lung fibrosis ${ }^{[41][42]}$.

We have to assume that this state of immune silencing is most likely occurring in around 2 billion people living in the helminth belt today. That this immune signature protects these people from the SARS-Covid19 and therefore explain the relatively low pervasiveness of the Covid-19 disease in the helminth belt can be shown through different means. We have chosen to show it graphically by comparing interactive global maps that indicate a clear negative correlation between helminth prevalence and Covid-19 infectivity and, importantly, mortality. More precise statistical analysis will be done in due time but for now, maps will serve just to illustrate the main arguments in the paper which are essentially based on our own personal interpretation of the previous scientific literature.

\section{COMMENTS ON THE FIGURES}

\section{Covid-19 deaths per million people.}

First, we present mapped data on the current world situation of the Covid-19 pandemic.

Epidemiological data in the media or in the literature are usually presented either as infection (confirmed by qPCR) by country, per million, or as absolute death numbers by country. We chose to compare the confirmed infection rate per $10^{5}$ from Google to our data consisting in deaths per million people by country. We think that deaths per million is a more accurate display of the crude data. And this for several reasons: Due to a lack of testing in many countries as well as the virus's ability to spread in people who don't show symptoms, counting the number of true infections turns out to be very difficult ${ }^{[43]}$. In addition, weather conditions can have an impact on the prevalence of respiratory diseases that have symptoms similar to COVID-19, likely influencing the number of tests performed on people with respiratory symptoms (the Russia case) ${ }^{[44]}$. For the same reason, Case Fatality Rates (deaths/confirmed infections) are not a valuable statistic since is depending upon the testing policy in each country, inducing a bias for lower rates in aggressively testing countries ${ }^{[45]}$ as shown in figure $1 \mathrm{~A}$, where Russia appears as a high prevalence country, in contrast to figure 1B, where "deaths per million" perhaps gives a more realistic picture of the current situation in that country. Finally, crude deaths 
per country, although an approximate measure is still not amenable to a true heat map for disease penetrance. In sum, we believe that death per million people is the closest to a fine approximation of disease prevalence/penetrance. One has to discount however some confounding factors that can influence the final death rate data. For instance, the Quality of the Health Care System of each country can obviously influence the disease outcome, or, on a more general and less quantifiable side, the political bias applied to death counting as we have recently seen ${ }^{[46]}$.

\section{Covid-19 map as a photo negative of global helminth infection prevalence.} In figure 2 we compared the death per million rate map (2A) with other maps representing the most recent available prevalence data for several helmith subspecies, including soil-transmitted helminths (2B), lymphatic filariasis (2C) and river blindness (caused by the nematode onchocerca volvulus) (2D). Also, as an example, we present the maps of two Caribbean countries that share a relatively long land border and have very different prevalences o both soil-transmitted diseases and lymphatic filariasis (Haiti high prevalence, Republican Republic low or very low). Importantly, the Covid-19 disease infection and death rates are also very disparate in those two countries, with a clear negative correlation between parasite occurrence and Covid-19 disease morbi/mortality ${ }^{[47]}$. The map panel also includes mapped data representing a proxy for helminth infection (the country sanitation status). To summarize this panel, we appreciate a strong correlation of low covi-19 morbi/mortality with high helminth prevalence. As expected, the one that more precisely overlaps with covid-19 is the map of soil-transmitted helminths and lymphatic filariasis as well as their probably closest proxy of soil-transmitted helminths, the map of sanitation status (whereby poor sanitation correlates with helminth infection) ${ }^{[48]}$.

\section{DISCUSSION}

In our previous report, we went thoroug hly across the biological events that most likely take place during the first days in the physiopathology of the coronavirus SARS. Among the key features, we mentioned the activation of a particular aspect of the innate immune system (the TLR7 receptor) as the more plausible centerpiece underpinning the hyperinflammatory state in the SARS-Covid-19 disease. By activating its natural intracellular receptor (TLR7), the SARS-CoV2 virus could trigger a massive unopposed inflammatory reaction within the lung. At the same time, the TLR7 activation, as described before, could drive the silencing of the natural counterbalance of the uncontrolled inflammation: the Th2 system ${ }^{[3]}$. 
In the present post, we show that the high parasite prevalence in the so called helminth belt could be of huge relevance to the way the Covid-19 disease is spreading and causing death accross the globe. Now we believe that, through the chronic infection of roughly 2 billion people living in the belt, infected individuals are adquiring a singular chronic immune predisposition that protects them from 1) being infected by the Covid-19 virus and 2) from suffering the lethal complications of this viral disease.

1. Resistance to infection by the SARS-Cov2 virus in the helminth belt: the eosinophilic link

The data are stubbornly telling us that Southern Asia and, especially Africa not only have lower death rates than the rest of the world, they also have less confirmed infections. Efforts have been made, particularly in Africa, to link the low morbi/mortality rates by Covid-19 to many possible influencers, among them, the median young age of the African population, population density variables, climate, etc ${ }^{[49][50][51]}$. All of them do not achieve statistical relevance by any means. Adding to the state of confusion, what people often forget is that Africa has rapidly become the number 1 continent in prevalence of hypertension in adults ${ }^{[52]}$. And, as we know, hypertension is the number 1 killer precondition in the Covid-19 pandemic ${ }^{[53]}$.

That people in the helminth belt could be protected from viral infection can be inferred from the high levels of eosinophils that are prevalent in those areas ${ }^{[54][55][56]}$. As we have shown before, due to their very specific payload of antiviral proteins, eosinophils are the best placed candidates to fill the role of first line anti-viral defense in the helminth belt. The role of eosinophils in the Covid-19 disease could be specially relevant since the SARSCoV2 virus, in contrast to the SARS-CoV1 of the 2003 outbreak, seems to be particularly adept at suppressing the first lines of defense ag ainst viruses. In fact, the SARS-CoV2 genome contains several open reading frames (ORF) that appear to be specialized in stifling various aspects of the body's anti-viral strategies. This includes blocking the Interferon response ${ }^{[57][58]}$ or decreasing the expression of the $\mathrm{MHCl}$ receptor which is essential for viral recognition by the CD8+ Cytotoxic $T$ Cells $(C T L)^{[59]}$. Eosinophils on the other side are known to kill viruses through different mechanisms including the already mentioned RNAses in the specific granules, the production of nitric oxide ${ }^{[60][61]}$ or the increased expression of host antiviral proteins like IRF-7, IFN-beta ${ }^{[62]}$ and MIP1alpha ${ }^{[63]}$.

2. When infected by the SARS-CoV2 virus, the Covid-19 disease is less lethal in the helminth belt: the Th2-IL-10 link 
The phenomenon of parasite-mediated immune suppression was first observed in the 1960s by Greenwood as a remarkably low prevalence of autoimmune diseases in Nigerian hospital admissions. Greenwood summarized his discovery as follows: "It is suggested that the infrequent occurrence of autoimmune disease in parts of tropical Africa is related to the immunological disturbance produced by multiple parasitic infections" ${ }^{\prime 64]}$.

Sixty years later, we know in every detail what Greenwood brilliantly observed and interpreted. In fact, helminth infection produces a whole array of effects, all of them leading to a state of basal low immune reactivity. This is best described by the so called Th2-IL-10 axis. IL-10 is an anti-inflammatory cytokine abundantly produced by the Th2 CD4+ lymphocytes. During helminth infection, it inhibits the activity of Th1 cells ${ }^{[65]}$ , suppresses the bidirectional crosstalk between NK cells and myeloid dendritic cells $(D C s)^{[66]}$, inhibits CD8+ cytotoxic $T$ cell function and directs macrophage polarization to an immunosuppressive phenotype ${ }^{[67]}$, all of which are needed for optimal pathogen clearance but can also contribute to tissue damage, specially when they get out of control ${ }^{[68]}$. Also, helminth infection is frequently associated with the expansion of regulatory T cells (Tregs). Tregs are a subset of TLRless T lymphocytes that patrol the body to assuage inflammation and avoid self-damage by an over-activated immune system ${ }^{[69]}$. Finally chronic helminth infection selectively populates the immune system with Th2 CD4+, Th9 CD4+ cells, and their effectors, Basophils, Mast Cells, and above all, Eosinophils ${ }^{[70][71]}$. All together, this showcases how helminths, over the ages, have designed a perfect ecological environment in which the survival of the host is probably as important as its own perpetuation. To summarize, chronic helminth infection produces a immune "dormant" state that is driven by the Th2-IL-10 axis that fits perfectly into its plan of maintaining the host alive (by preventing host's self-immune aggression), while suppressing the potential anti-parasite activity of the innate and adaptive immunity (including Th1 CD4+, CD8*, NK cells) ${ }^{[72]}$.

We, along with many others, have previously shown that what leads to the Acute Respiratory Failure in the Covid-19 disease is not the viral infection (i.e. the viral load) itself, but the extremely intense and seemingly uncontrolled inflammatory reaction as seen in the lungs of these patients ${ }^{[73]}$, and we all agree that aggressively countering this reaction is the obvious therapeutic approach to prevent the fatal outcome of the disease. In fact, immunosuppressants seem to have beneficial effects over the general outcome of the disease even when given as compassionate drugs in very severe cases $^{[74][75][76]}$. In an early paper, we have proposed the use of local 
immunosuppressants (i.e. through inhalers) early on during the course of the disease. We even suggested to start treatment as soon as the first flu like symptoms appear (particularly in groups of people at risk of developing serious complications) or even to administer the inhalers as a preventive measure in asymptomatic people living in very high risk environments like nursing homes (DOI: 10.5281/zenodo.3821979). The argument being that the sooner we start with the treatment the more efficient the immunosuppression will be before the cytokine storm starts shaping up in the lung's alveoli. By realizing that chronic infection with helminths does just that (to be able to face the disease with a muffled immune system), we now have come full circle.

To sum, we believe that chronic helminth infection provides infected people with an unexpected wealth of protective mechanisms against the Covid-19 disease and its lethal complications. This can be suitably articulated using a two legged model, whereby eosinophils, as abundantly present in helminth infected people, by preventing the viral replication in the epithelia, would represent the first leg. The other leg in this model, the Th2-IL-10 system, would be of extreme importance, since it would avert the building up of the stormy uncontrolled auto-immune reaction as seen in the lungs during SARS.

Figure 1. Comparative mapping of Covid-19 morbi/mortality A. Google map representing confirmed infection rates by a blue gradient (higher --> lower, intense blue --> light blue). B. Recent data are displayed in a heat map that shows countries with a low death rate due to covid-19 in green (light green for less than 4 deaths per million and deep green for less than 1 death per million people).

Figure 2. Maps of helminth prevalence compared to Covid-19 disease mortality rates

A. World map of Covid-19 disease in deaths per million (light green for less than 4 deaths per million and deep green for less than 1 death per million people). B. World map showing Prevalence of soil-transmitted helminths. C. World map of prevalence of lymphatic filariasis.

D. Onchocerciasis in Africa (Source: DALYs-WHO). E. Comparison of lymphatic filariasis endemism (E2) with Covid-19 death rates in Haiti vs Dominican Republic (E1). E. World map showing the degree of implementation of sanitation facilities by country.

Figure 3. Physiopatogenesis of the influence of helminth driven immune 
"silencing" on the viral infection rates and the odds of developing SARS

The lower part of the drawing describes the series of events that likely take place during the infection of the SARS-Cov2 virus on people at risk of developing SARS (hypertensive, diabetes, COPD, cancer). As described, this risk group is characterized by a basal chronic activation of the immune system ${ }^{[77][78]}$ that upon viral entry and replication will unfold the cytokine storm that is the trigger of SARS. At this point, the viral load will be irrelevant for the outcome of the disease.

The upper part depicts the most likely scenario in chronically helminth infected people, where the virus will first encounter a dense barrier of eosinophils. It is anticipated that after this encounter there will be a mild viral infection or no infection at all. Should some viral particles be able to replicate, the local alveolar innate immune system wouldn't present much fight with the neat consequence of a typical viral respiratory infection but, most importantly, without SARS.

A

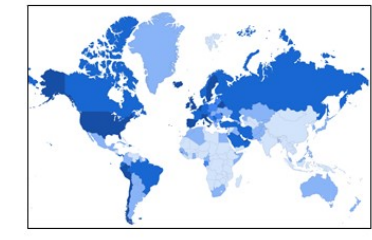

B

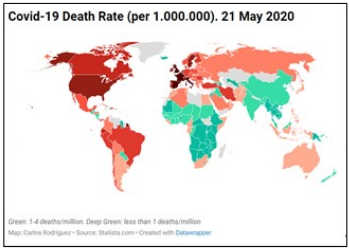

Figure 1

Figure 1 

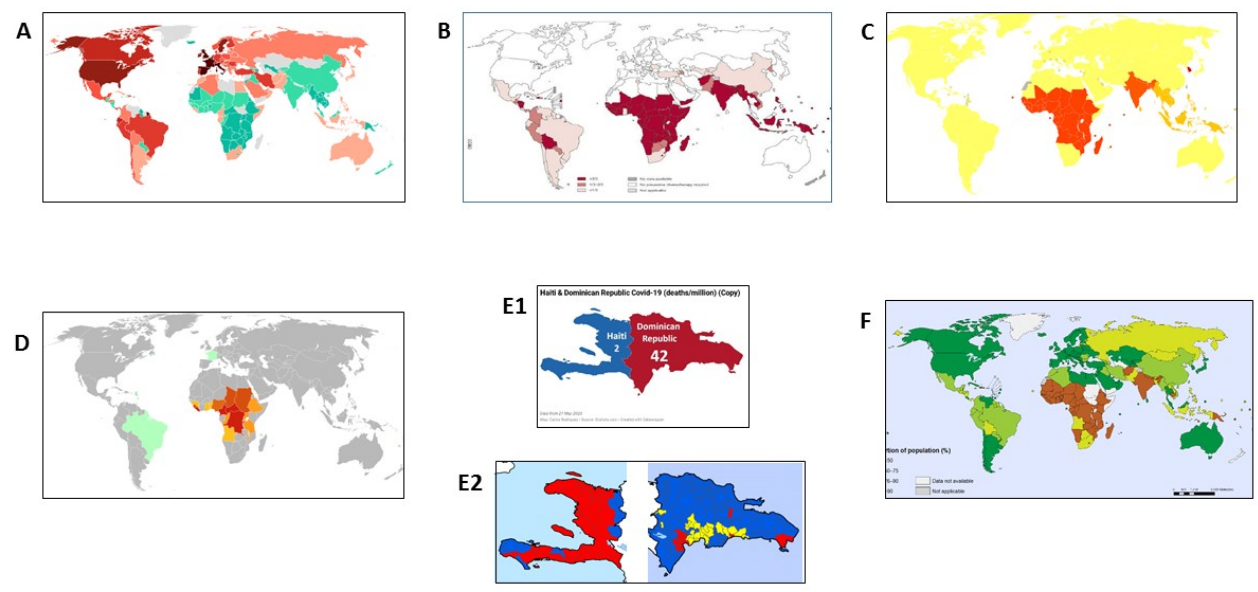

Figure 2

Figure 2

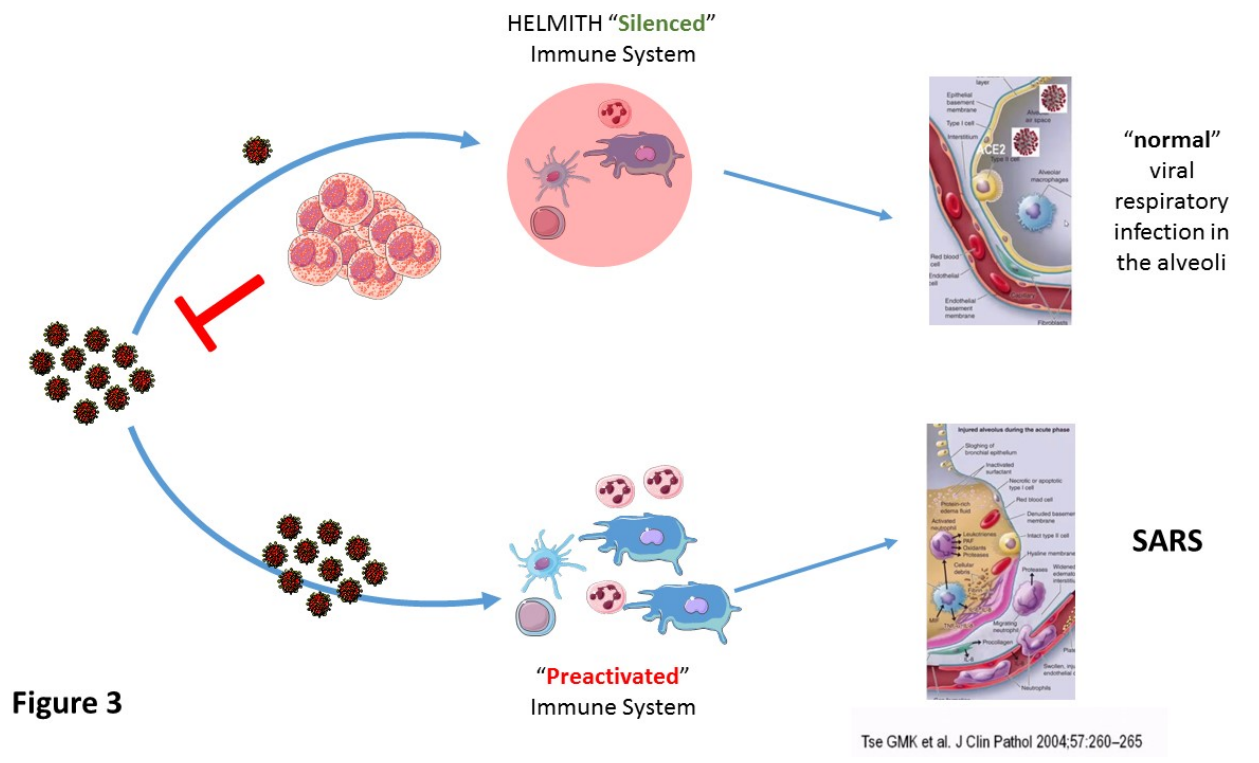

Figure 3

References

1. ^ Konstantinos Farsalinos, ANASTASIA BARBOUNI. (2020). Smoking, vaping and hospitalization for COVID-19. Qeios. doi:10.32388/26908a.

2. ' Makoto Miyara, Florence Tubach, Zahir Amoura. (2020). Low incidence of daily active tobacco smoking in patients with symptomatic COVID-19 infection. Qeios. doi:10.32388/WPP19W. 
3. ${ }^{a, b}$ Carlos Rodriguez, Carlos Veciana. (2020). ASTHMA AND COVID-19: THE EOSINOPHILIC LINK. Qeios. doi:10.32388/5/Y4IF.

4. `Jiyoung Yoon, Han-Na Um, Jinsun Jang, Young-An Bae, Woo-Jae Park, Hee Joo Kim. (2020). Corrigendum: Eosinophil Activation by Toll-Like Receptor 4 Ligands Regulates Macrophage Polarization. Front. Cell Dev. Biol., vol. 8. doi:10.3389/fcell.2020.00003.

5. ^Anupama Ariyaratne, Constance A. M. Finney. (2019). Eosinophils and Macrophages within the Th2-Induced Granuloma: Balancing Killing and Healing in a Tight Space. Infect Immun, vol. 87 (10). doi:10.1128/iai.00127-19.

6. ^ M. Yang, R. K. Kumar, P. M. Hansbro, P. S. Foster. (2012). Emerging roles of pulmonary macrophages in driving the development of severe asthma. Journal of Leukocyte Biology, vol. 91 (4), 557-569. doi:10.1189/jlb.0711357.

7. ^Marco Orecchioni, Yanal Ghosheh, Akula Bala Pramod, Klaus Ley. (2019). Macrophage Polarization: Different Gene Signatures in M1(LPS+) vs. Classically and M2(LPS-) vs. Alternatively Activated Macrophages. Front. Immunol., vol. 10. doi:10.3389/fimmu.2019.01084.

8. ^ Susan Stafford, Clifford Lowell, Sanjiv Sur, Rafeul Alam. (2002). Lyn Tyrosine Kinase Is Important for IL-5-Stimulated Eosinophil Differentiation. J Immunol, vol. 168 (4), 19781983. doi:10.4049/jimmunol.168.4.1978.

9. ^Alexander M. de Bruin, Miranda Buitenhuis, Koenraad F. van der Sluijs, Klaas P. J. M. van Gisbergen, Louis Boon, Martijn A. Nolte. (2010). Eosinophil differentiation in the bone marrow is inhibited by T cell-derived IFN-ץ. doi:10.1182/blood-2009-12-261339.

10. ^ Helene F. Rosenberg, Kimberly D. Dyer, Joseph B. Domachowske. (2008). Eosinophils and their interactions with respiratory virus pathogens. Immunol Res, vol. 43 (1-3), 128-137. doi:10.1007/s12026-008-8058-5.

11. ^Joseph B. Domachowske, Kimberly D. Dyer, Cynthia A. Bonville, Helene F. Rosenberg. (1998). Recombinant Human Eosinophit-Derived Neurotoxin/RNase 2 Functions as an Effective Antiviral Agent against Respiratory Syncytial Virus.J JNFECT DIS, vol. 177 (6), 1458-1464. doi:10.1086/515322.

12. ^ Valdirene S. Muniz, Peter F. Weller, Josiane S. Neves. (2012). Eosinophil crystalloid granules: structure, function, and beyond. Journal of Leukocyte Biology, vol. 92 (2), 281-288. doi:10.1189/jlb.0212067.

13. ^ Salahaddin Mahmudi-Azer, S.O. (Wole) Odemuyiwa, Redwan Moqbel. (2010). Intelligent granules: Are eosinophil crystalloid granules inimitable? Journal of Allergy and Clinical Immunology, vol. 126 (1), 28-30. doi:10.1016/j.jaci.2010.04.008.

14. ^ Maria T Rugeles, Charles M Trubey, Victoria I Bedoya, Ligia A Pinto, Joost J Oppenheim, Susanna M Rybak. (2003). Ribonuclease is partly responsible for the HIV-1 inhibitory effect activated by HLA alloantigen recognition. AIDS, vol. 17 (4), 481-486. 
doi:10.1097/00002030-200303070-00002.

15. ^ De Yang, Qian Chen, Helene F. Rosenberg, Susanna M. Rybak, Dianne L. Newton, Zhao Yuan Wang. (2004). Human Ribonuclease A Superfamily Members, EosinophilDerived Neurotoxin and Pancreatic Ribonuclease, Induce Dendritic Cell Maturation and Activation.J Immunol, vol. 173 (10), 6134-6142. doi:10.4049/jimmunol.173.10.6134.

16. ^ J.B. DOMACHOWSK, H.F. ROSENBERG. (2000). The role of eosinophils and their ribonucleases in respiratory viral infections. Respiratory Medicine, vol. 94 (10), 10121014. doi:10.1053/rmed.2000.0910.

17. ^ J. B. Domachowske, C. A. Bonville, K. D. Dyer, H. F. Rosenberg. (1998). Evolution of antiviral activity in the ribonuclease A gene superfamily: evidence for a specific interaction between eosinophil-derived neurotoxin (EDN/RNase 2) and respiratory syncytial virus. Nucleic Acids Research, vol. 26 (23), 5327-5332. doi:10.1093/nar/26.23.5327.

18. ^ Helene F. Rosenberg, Kimberly D. Dyer, Joseph B. Domachowske. (2008). Eosinophils and their interactions with respiratory virus pathogens. Immunol Res, vol. 43 (1-3), 128-137. doi:10.1007/s12026-008-8058-5.

19. ` James J. Lee, Helene F. Rosenberg. (2012). Eosinophils in Health and Disease. Elsevier.

20. ^James E. Wright, Marleen Werkman, Julia C. Dunn, Roy M. Anderson. (2018). Current epidemiological evidence for predisposition to high or low intensity human helminth infection: a systematic review. Parasites Vectors, vol. 11 (1). doi:10.1186/s13071-0182656-4.

21. ^ R. Muller. Epidemiological aspects of helminth infections.. doi:10.1079/9780851995168.0252.

22. `Martha Idalí Saboyá, Laura Catalá, Rubén Santiago Nicholls, Steven Kenyon Ault. (2013). Update on the Mapping of Prevalence and Intensity of Infection for SoilTransmitted Helminth Infections in Latin America and the Caribbean: A Call for Action. PLoS Neg/Trop Dis, vol. 7 (9), e2419. doi:10.1371/journal.pntd.0002419.

23. ^Rachel L Pullan, Jennifer L Smith, RashmiJasrasaria, SimonJ Brooker. (2014). Global numbers of infection and disease burden of soil transmitted helminth infections in 2010. Parasites \& Vectors, vol. 7 (1), 37. doi:10.1186/1756-3305-7-37.

24. ^ Mohamed S. Abdeltawabi, Nahla El Seddik, Hosni K. Salem. (2017). World Wide Epidemiology of Helminths Infection. doi:10.5772/67273.

25. ^Isabel Hagel, Neil R. Lynch, Mireya Pérez, Maria C. Di Prisco, Reina López, Edward Rojas. (1993). Relationship between the degree of poverty and the IgE response to Ascaris infection in slum children. Transactions of the Royal Society of Tropical Medicine and Hygiene, vol. 87 (1), 16-18. doi:10.1016/0035-9203(93)90401-b.

26. ^ (2006). Chapter 1. Investing in Health. doi:10.1596/978-0-8213-6179-5/chpt-1. 
27. ^ M. C. Freeman, A. N. Chard, B. Nikolay, J. V. Garn, C. Okoyo, J. Kihara. (2015). Associations between school- and household-level water, sanitation and hygiene conditions and soil-transmitted helminth infection among Kenyan school children. Parasites Vectors, vol. 8(1). doi:10.1186/s13071-015-1024- $x$.

28. ^ Sivaprakasam Rajasekaran, Rajamanickam Anuradha, Ramalingam Bethunaickan. (2017). TLR Specific Immune Responses against Helminth Infections. Journal of Parasitology Research, vol. 2017, 1-9. doi:10.1155/2017/6865789.

29. ^Stuart E. Turvey, David H. Broide. (2010). Innate immunity. Journal of Allergy and Clinical Immunology, vol. 125 (2), S24-S32. doi:10.1016/j.jaci.2009.07.016.

30. ^ Tom P. Monie. (2017). The Innate Immune System in Health and Disease. doi:10.1016/b978-0-12-804464-3.00006-5.

31. ^ Jinfang Zhu. (2015). T helper 2 (Th2) cell differentiation, type 2 innate lymphoid cell (ILC2) development and regulation of interleukin-4 (IL-4) and IL-13 production. Cytokine, vol. 75 (1), 14-24. doi:10.1016/j.cyto.2015.05.010.

32. a, b Bart Everts, Hermelijn H. Smits, Cornelis H. Hokke, Maria Yazdanbakhsh. (2010). Helminths and dendritic cells: Sensing and regulating via pattern recognition receptors, Th2 and Treg responses. Eur. J. Immunol., vol. 40 (6), 1525-1537. doi:10.1002/eji.200940109.

33. ^ Rick M. Maizels, Hermelijn H. Smits, Henry J. McSorley. (2018). Modulation of Host Immunity by Helminths: The Expanding Repertoire of Parasite Effector Molecules. Immunity, vol. 49 (5), 801-818. doi:10.1016/j.immuni.2018.10.016.

34. ^ Rick M. Maizels, Hermelijn H. Smits, Henry J. McSorley. (2018). Modulation of Host Immunity by Helminths: The Expanding Repertoire of Parasite Effector Molecules. Immunity, vol. 49 (5), 801-818. doi:10.1016/j.immuni.2018.10.016.

35. ^ GUOFENG CHENG, RONG LUO, CHAO HU, JIE CAO, YOUXIN JIN. (2013). Deep sequencing-based identification of pathogen-specific microRNAs in the plasma of rabbits infected with Schistosoma japonicum. Parasitology, vol. 140 (14), 1751-1761. doi:10.1017/s0031182013000917.

36. ^ Thomas Nutman. (2018). Faculty Opinions recommendation of Hookworm secreted extracellular vesicles interact with host cells and prevent inducible colitis in mice. doi:10.3410/f.733156179.793551825.

37. ^Henry J. McSorley, James P. Hewitson, Rick M. Maizels. (2013). Immunomodulation by helminth parasites: Defining mechanisms and mediators. International Journal for Parasitology, vol. 43 (3-4), 301-310. doi:10.1016/j.jppara.2012.11.011.

38. ${ }^{\wedge}, \ldots, \ldots$ (2019). Trichinella spiralis Excretory-Secretory Products Stimulate Host Regulatory T Cell Differentiation through Activating Dendritic Cells. Cells, vol. 8(11), 1404. doi:10.3390/cells8111404. 
39. `William Harnett, Margaret M. Harnett. (2001). Modulation of the host immune system by phosphorylcholine-containing glycoproteins secreted by parasitic filarial nematodes. Biochimica et Biophysica Acta (BBA) - Molecular Cell Research, vol. 1539 (1-2), 7-15. doi:10.1016/s0167-4889(01)00101-x.

40. ^ Lamyaa Al-Riyami, William Harnett. (2012). Immunomodulatory Properties of ES-62, a Phosphorylcholine - Containing Glycoprotein Secreted by Acanthocheilonema viteae. EMIDDT, vol. 12 (1), 45-52. doi:10.2174/187153012799278893.

41. ^ Colin J. Suckling, Shahabuddin Alam, Mark A. Olson, Kamal U. Saikh, Margaret M. Harnett, William Harnett. (2018). Small Molecule Analogues of the parasitic worm product ES-62 interact with the TIR domain of MyD88 to inhibit pro-inflammatory signalling. Sci Rep, vol. 8(1). doi:10.1038/s41598-018-20388-z.

42. `Colin J. Suckling, Sambuddho Mukherjee, Abedawn I. Khalaf, Ashwini Narayan, FraserJ. Scott, Sonal Khare. (2018). Synthetic analogues of the parasitic worm product ES-62 reduce disease development in in vivo models of lung fibrosis. Acta Tropica, vol. 185, 212-218. doi:10.1016/j.actatropica.2018.05.015.

43. 'Nidhi Subbaraman. (2020). Why daily death tolls have become unusually important in understanding the coronavirus pandemic. Nature. doi:10.1038/d41586-020-01008-1.

44. `Francois Cohen, Moritz Schwarz, Sihan Li, Yangsiyu Lu, Anant Jani. (2020). The Challenge of Using Epidemiological Case Count Data: The Example of Confirmed COVID-19 Cases and the Weather. doi:10.1101/2020.05.21.20108803.

45. ^Ned Stafford. (2020). Covid-19: Why Germany's case fatality rate seems so low. BMJ. doi:10.1136/bmj.m1395.

46. ^Helcio R.B. Orlande, Marcelo Colaco, George S. Dulikravich, Luiz F.S. Ferreira. (2020). Estimation Of State Variables And Model Parameters For The Evolution Of COVID-19 In The City Of Rio de Janeiro. doi:10.1101/2020.05.21.20108407.

47. ^Martha Idalí Saboyá, Laura Catalá, Rubén Santiago Nicholls, Steven Kenyon Ault. (2013). Update on the Mapping of Prevalence and Intensity of Infection for SoilTransmitted Helminth Infections in Latin America and the Caribbean: A Call for Action. PLoS Neg/Trop Dis, vol. 7 (9), e2419. doi:10.1371/journal.pntd.0002419.

48. ^ Kathrin Ziegelbauer, Benjamin Speich, DanielMäusezahl, Robert Bos, Jennifer Keiser, Jürg Utzinger. (2012). Effect of Sanitation on Soil-Transmitted Helminth Infection: Systematic Review and Meta-Analysis. PLoS Med, vol. 9 (1), e1001162. doi:10.1371/journal.pmed.1001162.

49. `Mohammad Alamgir Hossain. (2020). Is the spread of COVID-19 across countries influenced by environmental, economic and social factors?. doi:10.1101/2020.04.08.20058164.

50. `Carl Andrew Pearson, Cari Van Schalkwyk, Anna M Foss, Kathleen M O'Reilly, Juliet R C 
Pulliam, CMMID COVID-19 working group. (2020). Projected early spread of COVID-19 in Africa. doi:10.1101/2020.04.05.20054403.

51. ^Ikpe Akpan, Izuchukwu C. Ezeume, Atinuke Ezeume, Asuama Akpan, Esther Justice Akpand, Elias Udomboso. (2020). Controlling the Spread of COVID-19 Pandemic: Can West Africa Weather the Storm?. SSRN Journal. doi:10.2139/ssrn.3580473.

52. ^ William Kofi Bosu, Siobhan Theresa Reilly, Justice Moses Kwaku Aheto, Eugenio Zucchelli. (2019). Hypertension in older adults in Africa: A systematic review and metaanalysis. PLOS ONE, vol. 14 (4), e0214934. doi:10.1371/journal.pone.0214934.

53. ^Bryan Williams, Yi Zhang. (2020). Hypertension, renin-angiotensin-aldosterone system inhibition, and COVID-19. The Lancet. doi:10.1016/s0140-6736(20)31131-4.

54. ^Ayodele Olushola Ilesanmi, GinnikachiJennifer Ekwe, Rosemary Isioma Ilesanmi, Damilola Temitope Ogundele, Jacob Kehinde Akintunde, Oluwasogo Adewole Olalubi. (2016). Prevalence of latent eosinophilia among occupational gardeners at Babcock University, Nigeria. Asian Pacific Journal of Tropical Biomedicine, vol. 6 (6), 511-515. doi:10.1016/j.apjtb.2015.12.022.

55. ^ D Ray, R Abel, K G Selvaraj. (1993). Epidemiology of pulmonary eosinophilia in rural south India--a prospective study, 1981-86.. Journal of Epidemiology \& Community Health, vol. 47 (6), 469-474. doi:10.1136/jech.47.6.469.

56. ^ Neha Thakur, Narendra Rai. (2016). Alarmingly High Incidence of Eosinophilia in Barabanki and Neighboring Districts of Eastern Uttar Pradesh: A Prospective HospitalBased Study.J Trop Pediatr. doi:10.1093/tropej/fmw035.

57. ^ Sarah A. Kopecky-Bromberg, Luis Martinez-Sobrido, Matthew Frieman, Ralph A. Baric, Peter Palese. (2006). Severe Acute Respiratory Syndrome Coronavirus Open Reading Frame (ORF) 3b, ORF6, and Nucleocapsid Proteins Function as Interferon Antagonists. NI, vol. 81 (2), 548-557. doi:10.1128/jvi.01782-06.

58. ^ Veljko Veljkovic, Slobodan Paessler. (2020). COVID-19 Orf3b protein: the putative biological function and the therapeutic target. doi:10.21203/rs.2.24483/v1.

59. ^ Yiwen Zhang, Junsong Zhang, Yingshi Chen, Baohong Luo, Yaochang Yuan, Feng Huang. (2020). The ORF8 Protein of SARS-CoV-2 Mediates Immune Evasion through Potently Downregulating MHC-I. doi:10.1101/2020.05.24.111823.

60. ^ Matthew G. Drake, Elizabeth R. Bivins-Smith, Becky J. Proskocil, Zhenying Nie, Gregory D. Scott, James J. Lee. (2016). Human and Mouse Eosinophils Have Antiviral Activity against Parainfluenza Virus. Am J Respir Cell Mol Biol, vol. 55 (3), 387-394. doi:10.1165/rcmb.2015-0405oc.

61. ^ Matthew Drake, Becky Proskocil, Zhenying Nie, Fryer Allison, Jacoby David. (2016). Human eosinophil toll-like receptor 7 -induced nitric oxide is antiviral against parainfluenza. doi:10.1183/13993003.congress-2016.pa3970. 
62. ^Simon Phipps, Chuan En Lam, Suresh Mahalingam, Matthew Newhouse, Ruben Ramirez, Helene F. Rosenberg. (2007). Eosinophils contribute to innate antiviral immunity and promote clearance of respiratory syncytial virus. doi:10.1182/blood2007-01-071340.

63. ^Joseph B. Domachowske, Cynthia A. Bonville, Kimberly D. Dyer, Andrew J. Easton,

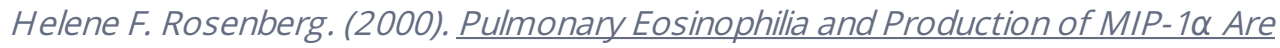
Prominent Responses to Infection with Pneumonia Virus of Mice. Cellular Immunology, vol. 200 (2), 98-104. doi:10.1006/cimm.2000.1620.

64. ^ B.M. Greenwood. (1968). AUTOIMMUNE DISEASE AND PARASITIC INFECTIONS IN NIGERIANS. The Lancet, vol. 292 (7564), 380-382. doi:10.1016/s0140-6736(68)905953.

65. ^ Giorgio Trinchieri. (2007). Interleukin-10 production by effector T cells: Th1 cells show self control. doi:10.1084/jem.20070104.

66. ^ Sanja Mandaric, Senta M. Walton, Thomas Rülicke, Kirsten Richter, Mathilde J. H. Girard-Madoux, Björn E. Clausen. (2012). IL-10 Suppression of NKIDC Crosstalk Leads to Poor Priming of MCMV-Specific CD4 T Cells and Prolonged MCMV Persistence. PLoS Pathog, vol. 8(8), e1002846. doi:10.1371/journal.ppat.1002846.

67. `Yishan Chuang, Michelle E Hung, Brianne K Cangelose, Joshua N Leonard. (2016). Regulation of the IL-10-driven macrophage phenotype under incoherent stimuli. Innate Immun, vol. 22 (8), 647-657. doi:10.1177/1753425916668243.

68. ^Kevin N. Couper, Daniel G. Blount, Eleanor M. Riley. (2008). IL-10: The Master Regulator of Immunity to Infection. J Immunol, vol. 180 (9), 5771-5777. doi:10.4049/jimmunol.180.9.5771.

69. ^Jayden Logan, Severine Navarro, Alex Loukas, Paul Giacomin. (2018). Helminthinduced regulatory $T$ cells and suppression of allergic responses. Current Opinion in Immunology, vol. 54, 1-6. doi:10.1016/j.coi.2018.05.007.

70. ^Rajamanickam Anuradha, Saravanan Munisankar, Yukthi Bhootra, Jeeva Jagannathan, Chandrakumar Dolla, Paul Kumaran. (2016). IL-10-and T GFß-mediated Th9 Responses in a Human Helminth Infection. PLoS Neg/Trop Dis, vol. 10 (1), e0004317. doi:10.1371/journal.pntd.0004317.

71. ^M.E.J. Woolhouse. (1993). A theoretical framework for immune responses and predisposition to helminth infection. doi:10.1111/pim.1993.15.10.583.

72. ^David L Sacks. (2002). Faculty Opinions recommendation of IL-10 is critical for host resistance and survival during gastrointestinal helminth infection.. doi:10.3410/f.1005298.62309.

73. ^ Ricardo JJose, Ari Manuel. (2020). COVID-19 cytokine storm: the interplay between inflammation and coagulation. The Lancet Respiratory Medicine. doi:10.1016/s2213- 
2600(20)30216-2.

74. `Xiaoling Xu, Mingfeng Han, Tiantian Li, Wei Sun, Dongsheng Wang, Binqing Fu. (2020). Effective treatment of severe COVID-19 patients with tocilizumab. Proc NatI Acad Sci USA, vol. 117 (20), 10970-10975. doi:10.1073/pnas.2005615117.

75. ${ }^{\wedge}$, , . Severe Coronavirus disease 2019 (COVID-19). doi:10.1148/cases.20201281.

76. ^ Srinivasa Reddy Bonam, Srini V. Kaveri, Anavaj Sakuntabhai, Laurent Gilardin, Jagadeesh Bayry. (2020). Adjunct Immunotherapies for the Management of Severely IIICOVID-19 Patients. Cell Reports Medicine, vol. 1 (2), 100016. doi:10.1016/j.xcrm.2020.100016.

77. ^ Madhu V. Singh, Mark W. Chapleau, Sailesh C. Harwani, Francois M. Abboud. (2014). The immune system and hypertension. Immunol Res, vol. 59 (1-3), 243-253. doi:10.1007/s12026-014-8548-6.

78. ^ Bruce Bartholow Duncan, Maria Inês Schmidt. (2001). Chronic activation of the innate immune system may underlie the metabolic syndrome. Sao Paulo Med. J., vol. 119 (3), 122-127. doi:10.1590/s1516-31802001000300008. 